\title{
Estrategias didácticas para el desarrollo exitoso de la innovación y el emprendimiento en la educación superior
}

\author{
Ramos Sánchez, Erick \\ Universidad Internacional de Ciencia y Tecnología (UNICyT) \\ Panamá, Panamá \\ ORCID: 0000-0002-1173-0143 \\ López de Ramos, Aura \\ Universidad Internacional de Ciencia y Tecnología (UNICYT) \\ Panamá, Panamá \\ ORICID: 0000-0002-8983-9704
}

\begin{abstract}
The Covid-19 pandemic has prompted the need to offer more effective training in innovation and entrepreneurship as part of the solution to the great global and persistent problem of unemployment. The objective of this research was to formulate didactic strategies used in basic and specialized undergraduate and graduate courses for the development of meaningful learning experiences. The research was based on the theory of the systematization of practical experiences. The didactic strategies used by the course teachers who have attended 261 students throughout the 12 academic periods in which this subject has been offered to the 18 careers offered by the institution at the Bachelor level were identified and isolated. The pedagogical model used by the institution is Flipped Learning (blended or virtual modality). It was found that the strategies that the teacher must include to promote a creative environment are: 1 . Flexible planning, 2. Conceptual adaptation, 3. Relaxed and rewarding environment for students, 4. Participative and interactive roles, 5. Productivity or personal fulfillment, 6. Student satisfaction and self-learning awareness.
\end{abstract}

Keywords: Didactic strategies, Flipped learning, Innovation in times of crisis, Innovation and entrepreneurship, Systematization of experience.

\section{Resumen}

La pandemia por Covid-19 ha impulsado la necesidad de ofrecer una formación más efectiva en innovación y emprendimiento como parte de la solución al gran problema 
mundial y persistente del desempleo. El objetivo de esta investigación fue formular estrategias didácticas usadas en cursos básicos y especializados de pregrado y grado para el desarrollo de experiencias de aprendizaje significativas. La investigación se cimentó en la teoría de la sistematización de experiencias prácticas. Se identificaron y aislaron las estrategias didácticas usadas por los profesores del curso que han atendido a 261 estudiantes a lo largo de 12 períodos académicos en que se ha ofrecido esta asignatura a las 18 carreras que ofrece la institución a nivel de Licenciaturas. El modelo pedagógico usado por la institución es el de Flipped Learning (modalidad semipresencial o virtual). Se encontró que las estrategias que el docente debe incluir para favorecer un ambiente creativo son: 1. Planificación flexible, 2. Adaptación conceptual, 3. Clima distendido y gratificante para los estudiantes, 4. Roles participativos e interactivos, 5. Productividad o realización personal, 6 . Satisfacción discente y conciencia de autoaprendizaje.

Palabras clave: Estrategias didácticas, Flipped learning, Innovación en tiempos de crisis, Innovación y emprendimiento, Sistematización de experiencia.

\section{INTRODUCCIÓN}

La creatividad y la innovación generalmente son consideradas cualidades gemelas y son cualidades muy apreciadas por parte de las empresas y muchas organizaciones [1]. No obstante, son dos conceptos diferentes y que se pueden confundir. La creatividad es la capacidad de un individuo para generar nuevas ideas o conceptos, por otro lado, la innovación se refiere a la capacidad de implementar algo nuevo o modificar elementos ya existentes con el fin de mejorarlos. Ambas son competencias fundamentales para el éxito de una empresa. Se puede afirmar que las empresas que no esgrimen creatividad e innovación tienden a desaparecer.

En tal sentido, como competencias, ambas deben ser desarrolladas por los colaboradores de la empresa, así como por los directivos y demás personal, tanto operativo como administrativo, todos alineados por una misma Misión y Visión. La sociedad espera de la academia el desarrollo de estas competencias en los jóvenes estudiantes, mientras, a más de un año de la declaración de la pandemia por Covid-19 por parte de la OMS, persiste y se agrava el problema de desempleo a nivel mundial y particularmente en la región de Centroamérica. Es por ello por lo que se presenta la necesidad de buscar y encontrar nuevas formas de impulsar el desarrollo de más y mejores fuentes de empleo. Es cada vez más importante que los futuros profesionales culminen sus carreras para emprender, innovar y resolver los problemas de la sociedad, antes que dirigirse a las empresas y organizaciones 
con sus títulos y licencias a buscar trabajos.

En la llamada "Nueva Normalidad" se hace evidente la necesidad de que los profesionales dominen las competencias de la innovación, el emprendimiento y la solución de problemas, como garantía de sustento de una sociedad en una nueva crisis económica por la pérdida de empleos y organizaciones, pero, también por la afectación de muchas de las que permanecen con algún nivel de actividad. En tal sentido, se requiere un nuevo profesional capaz de reinventar la sociedad del conocimiento en esa "Nueva Normalidad. En este trabajo se presentan los resultados de la sistematización de la experiencia obtenida en el curso denominado Design Thinking, StartUp y Lean Canvas que forma parte del plan de estudios de las carreras de licenciatura de la Universidad Internacional de Ciencia y Tecnología (UNICYT).

\section{MÉTODO}

La investigación está basada en la sistematización de experiencias prácticas, como actividad de producción de conocimientos sobre la práctica docente, con el propósito de generar retroalimentación de sus aconteceres y verterlos nuevamente en la experiencia para rectificar cualquier falla, reforzar y replicar lo más significativo de los aprendizajes.

El Dr. Carlos Villalva Heredia y su equipo afirman que, "la sistematización de experiencias representa, actualmente, una vía de recuperación, examen y asimilación de las prácticas educativas del sistema académico que facilita la comprensión de ambientes, así como el sentido, la esencia, las lógicas y los elementos problemáticos que muestran tales prácticas. [2] Por su parte, Jennifer Cano y John García indican que la sistematización de la experiencia se identifica con el enfoque epistemológico dialógico e interactivo ya que se desarrollan espacios de interacción, comunicación y de relación con los estudiantes gracias a las prácticas realizadas durante la metodología de enseñanza.[3]

Convencidos de la necesidad de producir profesionales con las competencias de innovación y emprendimiento requeridas por una sociedad ávida de puestos de trabajo y profesionales de las nuevas generaciones capaces de resolver los actuales problemas y retos, incluso aquellos aun no detectados, se identificaron y aislaron como soporte transversal de la práctica, las estrategias didácticas aplicadas por los docentes en los cursos de la asignatura Design Thinking, Startup y Lean Canvas (DTSLC) para el desarrollo exitoso del emprendimiento de ideas innovadoras.

Las prácticas consideradas en esta sistematización se fraccionaron de acuerdo con las etapas o reiteraciones de la aplicación del método para emprender Design Thinking, con flexibilidad, considerando que algunas ideas requieren más reiteraciones o ciclos que otras para encontrar el balance necesario para un prototipo viable. Ello permitió establecer una línea de tiempo. 
La población para este estudio lo constituyó 261 estudiantes, que cursaron la asignatura DTSLC, durante una docena de periodos académicos en que se ha ofrecido esta asignatura a las 18 carreras que ofrece la institución a nivel de licenciaturas. No se consideró el cálculo de una muestra ya que fueron considerados todos los estudiantes de la población. Los datos fueron obtenidos de los registros académicos de los cursos y se constataron con los registros anecdóticos de los profesores que dictaron el curso DTSLC. La revisión de literatura, artículos y libros sobre el tema de la innovación y el emprendimiento, así como de la enseñanza de dichas competencias permitieron precisar cómo este trabajo de investigación contribuye con la comprensión de la importancia de la adquisición de las competencias en innovación y emprendimiento para los estudiantes de educación superior. [4], [5], [6], [7], [8]

\section{RESULTADOS}

Se identificaron y aislaron las estrategias didácticas usadas por los profesores del curso que han atendido a 261 estudiantes a lo largo de 12 períodos académicos en que se ha ofrecido esta asignatura a las 18 carreras que ofrece la institución a nivel de Licenciaturas (Fig. 1).

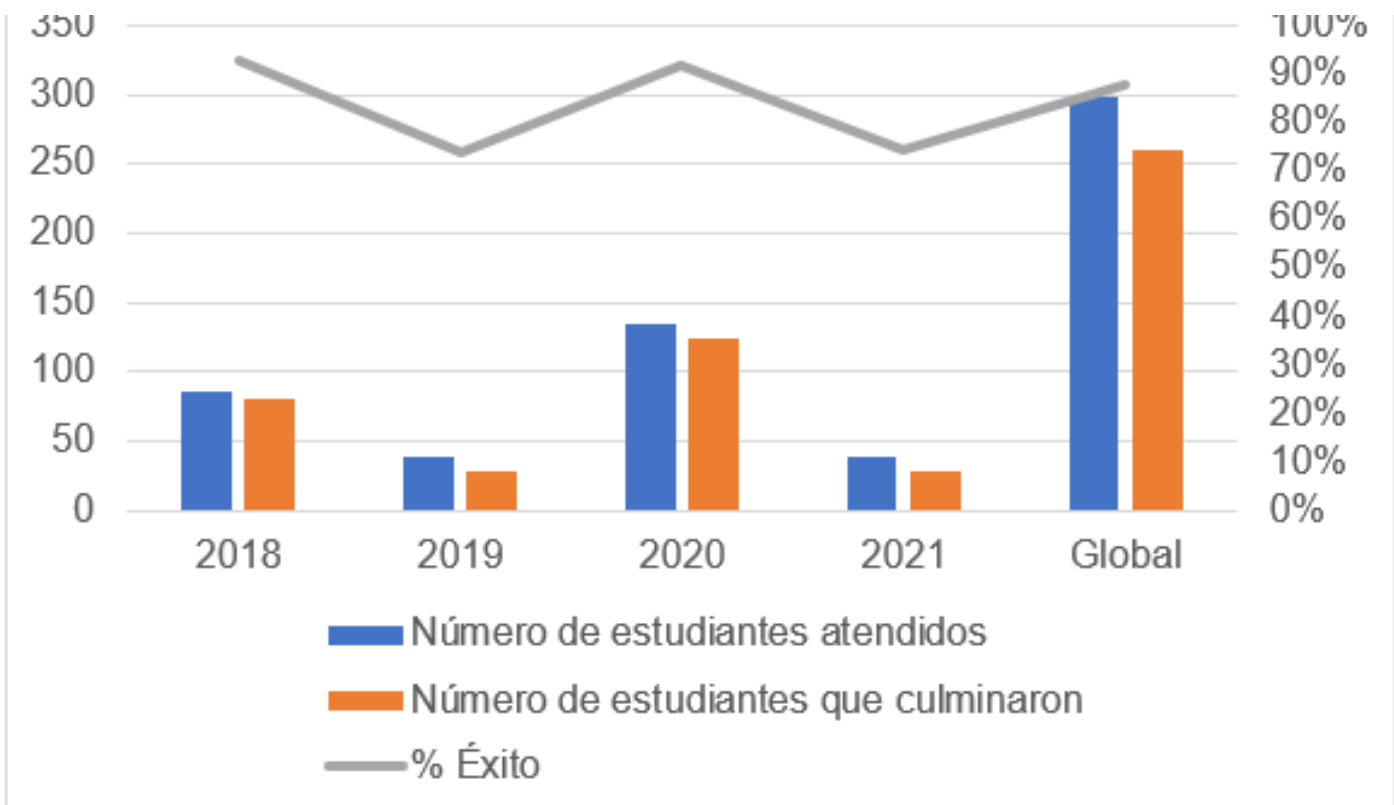

Fig. 1. Estadísticas de los estudiantes atendidos, estudiantes que culminaron su emprendimiento y las cifras totales. El año 2021 incluye datos solo hasta el mes de mayo.

En el año 2018 se dio por primera vez la asignatura de Design Thinking Startup y Lean Canvas e incluyó a estudiantes de diferentes promociones, en el año 2019 cursaron estudiante exclusivamente de esa promoción. Ya en el 2020, a pesar de la pandemia, se pudo poner en funcionamiento al 100\% el programa. Las cifras reportadas en el año 2021 son menores cuando se compararan con el año anterior, ya que solo incluyen los resultados del primer 
cuatrimestre. El porcentaje global de éxito (estudiantes que logran concluir el modelo de negocio de su idea innovadora) es del $88 \%$. Aunque no hay un estudio sobre el $12 \%$ de fracasos, el registro anecdótico de los profesores da cuenta de que la mayoría de los casos se debe a deserciones entre el inicio y la mitad del periodo académico, ocasionadas por la toma de decisión del estudiante motivado a la suspensión temporal o definitiva de sus estudios debido razones financieras (nueva oferta o pérdida de empleo) o de salud (enfermedad de un familiar que requiere tiempo de atención por parte de estudiante o condiciones de salud propias que le impiden proseguir sus estudios).

El modelo pedagógico usado por la institución es el de Flipped Learning (modalidad semipresencial o virtual con sesiones de encuentros virtuales y síncronos). Se encontró que las estrategias que el docente debe incluir, pues favorecen el ambiente creativo que es esencial para este tipo de asignaturas, son: 1. Planificación flexible, 2. Adaptación conceptual, 3. Clima distendido y gratificante para los estudiantes, 4. Roles participativos e interactivos, 5. Productividad o realización personal, 6. Satisfacción discente y conciencia de autoaprendizaje.

En tal sentido, la planificación flexible se convierte en un factor crítico a la hora de innovar y emprender. Los estudiantes se encuentran, en estos tiempos de pandemia, ansiosos por salir de esta situación y, por ello, presionar por el cumplimiento de los ciclos o etapas, lejos de ayudar a alcanzar sus objetivos, los lleva a descuidar los detalles que hacen de su idea innovadora un emprendimiento viable y atractivo. Es bien conocido que cada estudiante aprende a su propio ritmo y que, por su parte, cada proyecto es distinto y requiere tiempos distintos para cada ciclo. El profesor innovador y creativo posee una disposición flexible hacia las personas, las decisiones y los acontecimientos; no solo tolera los cambios, sino que está abierto a ellos. Además, investigaciones en el campo de la psicología conductual han identificado rasgos personales asociados con la creatividad que incluyen: flexibilidad, mentalidad abierta, tolerancia a la ambigüedad, toma de riesgos y voluntad de jugar e improvisar. [9]

Por otro lado, los roles participativos e interactivos y la productividad dependen en gran medida de clima distendido y gratificante en el que la creatividad fluya sin limitaciones comunes o estereotipos propios de la gestión clásica del mercado. También es importante que los emprendedores cuenten con los conocimientos, habilidades y actitudes que les permitan gestionar con éxito una iniciativa empresarial. Es por ello por lo que las prácticas de gestión empresarial, a través del liderazgo y la planeación estratégica, juegan un papel fundamental en el emprendimiento y desarrollo de una iniciativa empresarial. [10] Las estrategias didácticas usadas por los docentes fueron esencialmente una integración de concepciones tecnologías, de la ciencia y del arte, pero con énfasis en la tecnología, debido a la influencia de las medidas tomadas por el estado para proteger a la población 
del contagio por la pandemia. En tal sentido, el uso de las videoconferencias y herramientas colaborativas formaron parte importante de los cursos en el último año. Los trabajos de innovación o emprendimiento desarrollados por los estudiantes se denominaron ideas creativas y fueron presentadas en las 5 ferias de emprendimiento (UNICyTIOS: UNICYT Incubator of StartUps).

Inicialmente, los estudiantes comienzan desarrollando o reforzando la empatía con el cliente o usuario, reconociendo, los sesgos cognitivos, la innovación, el emprendimiento $y$, en general, las habilidades, conocimientos y actitudes para abordar un reto. Se destaca el trabajo colaborativo y se forman distintos niveles de equipos (dos participantes, tres participantes, más de tres participantes y el nivel de todo el curso). Estos niveles de equipos permiten a los estudiantes manejar con mayor facilidad las iteraciones de las versiones de su idea y acumular más y nuevos complementos para producir una idea innovadora y competitiva. Esto es lo que permite viabilizar, técnica y económicamente, una solución a la necesidad del cliente o usuario a través de la metodología Design Thinking, Esto es cónsono con lo que refiere Tim Brown en su publicación, cuando afirma que: Antes de la mitad del siglo $X X$, el diseño fue tratado como un paso posterior en el proceso de desarrollo, donde los diseñadores no involucrados, ajenos a las etapas principales del desarrollo, proveyeron a las ideas (productos y servicios) de excelentes elementos que mejoraron la estética haciéndolos más atractivos. Después de la segunda mitad del siglo XX, el diseño fue cada vez más competitivo y valioso, incorporando electrónica de consumo, la automatización, centrados en la demanda de los compradores y usuarios. [11]

Luego, los estudiantes deben formular, mediante la práctica de la empresa emergente (Startup) y del uso de las herramientas Business Model Canvas, o Lienzo del Modelo de Negocio, su propio modelo de negocios con sus ideas innovadoras. El Modelo Lean Canvas es un método para describir, visualizar, evaluar y modificar modelos de negocio, atribuido a Alex Osterwalder. [12]

\section{CONCLUSIONES}

Las estrategias didácticas aplicadas a los cursos de innovación y emprendimiento "Design Thinking, Startup y Lean Canvas" han logrado un buen resultado, con una efectividad global en cuanto al número de emprendimientos finalizados por estudiante inscrito, del $88 \%$. Estas estrategias son: 1. Planificación flexible, 2. Adaptación conceptual, 3. Clima distendido y gratificante para los estudiantes, 4. Roles participativos e interactivos, 5. Productividad o realización personal, 6 . Satisfacción discente y conciencia de autoaprendizaje. 


\section{Referencia}

[1] E. Córdoba Zuñiga, J. Castillo Jency, y N. Castillo Quesada, “Creatividad e Innovación: Motores de Desarrollo Empresarial." Lámpsakos, no. 19, pp. 55-65, enero 2018. https://doi. org/10.21501/21454086.2663

[2] C. Villalva, L. Niama, L. Silva, K. Carrera, M. Moreno, y M. Herrera, "La sistematización de experiencias y para qué nos sirve en la Educación Superior Tercermundista." Revista Dilemas Contemporaneos: Educación, Politica y Valores, vol. 6, no. 2, pp. 1-19, enero 2019. http://search. ebscohost.com/login.aspx?direct=true\&db=fap\&AN=134365766\&lang=es\&site=ehost-live

[3] J. A. Cano-Guevara y J. A. García-Quintero, "Flipped Classroom en la enseñanza de lógica y algoritmos en la universidad de la amazonia; una sistematización de experiencias", Rev. Cient., vol. 26, pp. 53-61, oct. 2016. https://doi.org/10.14483/23448350.11090

[4] M. E. Pensado Fernández y J. M. Ortiz García, "Emprendimiento como dimensión de formación en estudiantes universitarios. Un análisis curricular en el caso de la UV", Revista Ciencia Administrativa, no. 2, pp. 162-173, julio 2018. https://www.uv.mx/iiesca/files/2019/02/18CA201802.pdf

[5] E. Aldana, I. Gil, M. Ortiz, J. Tafur, y P. Castañeda, "Gestión Educativa Hacia El Desarrollo De Competencias De Emprendimiento En Estudiantes Universitarios", Revista Orbis, vol. 16, pp. 85-95, julio 2020. https://dialnet.unirioja.es/servlet/articulo?codigo $=7611498$

[6] C. Vélez, M. A. Bustamante, B. A. Loor, y S. M. Afcha, "La educación para el emprendimiento como predictor de una intención emprendedora de estudiantes universitarios", vol. 13, no. 2, Formación Universitaria, pp. 63-71, febrero 2020. https://doi.org/10.4067/S0718-50062020000200063

[7] P. E. Vargas, "Modelo decisional para diseño de políticas públicas para el emprendimiento en base al GEM Panamá", en Actas del V Congreso de Investigación, Desarrollo e Innovación de la Universidad Internacional de Ciencia y tecnología - IDI-UNICyT 2020, pp. 409-431. https://doi. org/10.47300/978-9962-5599-8-6

[8] P. E. Vargas, y M. del Cid, “Análisis reflexivo sobre filosofía práctica y emprendimiento”, en Actas del V Congreso de Investigación, Desarrollo e Innovación de la Universidad Internacional de Ciencia y tecnología - IDI-UNICyT 2020, pp. 431-438. https://doi.org/10.47300/978-9962-5599-8-6

[9] R. Mehta, D. Henriksen, y J. M. Rosenberg, "It's not about the tools: Ed-tech training needs to go beyond specific tools and instead enable teachers with an adaptable, creative mindset", Educational Leadership, vol. 76, no. 5, pp. 64-69, february 2019. http://danah-henriksen.com/wp-content/ uploads/2019/03/Educational-Leadership-CFTF-mindset.pdf

[10] A. López-Lemus, y M. T. de la Garza Carranza, “Las prácticas de gestión empresarial, innovación y emprendimiento: Factores influyentes en el rendimiento de las firmas emprendedoras", Nova Scientia, vol. 11, no. 22, enero 2019, pp. 357-383, https://doi.org/10.21640/ns.v11i22.1795

[11] T. Brown, "Design Thinking", Harvard Business Review, pp. 3-10, junio 2008. https://readings. design/ PDF/Tim\%20Brown,\%20Design\%20Thinking.pdf

[12] A. Osterwalder, Y. Pigneur, A. Smith,y F. Etiemble, The Invincible Company: How to Constantly Reinvent Your Organization with Inspiration From the World's Best Business Models, New Jersey, USA: John Wiley \& Sons, 2020. 


\section{Autorización y Licencia CC}

Los autores autorizan a APANAC XVIII a publicar el artículo en las actas de la conferencia en Acceso Abierto (Open Access) en diversos formatos digitales (PDF, HTML, EPUB) e integrarlos en diversas plataformas online como repositorios y bases de datos bajo la licencia CC:

Attribution-NonCommercial-ShareAlike 4.0 International (CC BY-NC-SA 4.0) https://creativecommons. org/licenses/by-nc-sa/4.0/.

Ni APANAC XVIII ni los editores son responsables ni del contenido ni de las implicaciones de lo expresado en el artículo. 